\title{
GW23-e2639 ATORVASTATIN IN PULMONARY HYPERTENSION (APATH) STUDY
}

doi:10.1136/heartjnl-2012-302920a.254

He Jianguo, Zeng Weijie. Fuwai Hospital

Objectives Statins have been shown to both prevent and attenuate pulmonary hypertension in animal models. This study investigates the potential therapeutic benefits of atorvastatin as an affordable treatment for pulmonary hypertension patients. Two hundred and twenty patients with pulmonary arterial hypertension (PAH) or chronic thromboembolic pulmonary hypertension (CTEPH) were randomised, double-blind, to receive atrovastatin $10 \mathrm{mg}$ daily or matching placebo in addition to supportive care. At 6 months, 6-min walk distance decreased by $16.6 \mathrm{~m}$ in the atorvastatin group and $14.1 \mathrm{~m}$ in the placebo group. The mean placebo-corrected treatment effect was $-2.5 \mathrm{~m}$ ( $95 \% \mathrm{CI}-38$ to $33 ; \mathrm{p}=1.0)$, based on intention to treat. A small non-significant increase in pulmonary vascular resistance and fall in cardiac output was seen in both treatment groups. There was no significant difference in the proportion of patients who improved, remained stable or showed deterioration in WHO functional class between atorvastatin and placebo treatments. Nine patients died in the atorvastatin group and 11 in the placebo group. Serum cholesterol levels fell significantly on atorvastatin treatment. Discontinuation rates were $23.2 \%$ and $26.9 \%$ on atorvastatin and placebo respectively. Atorvastatin $10 \mathrm{mg}$ daily has no beneficial effect on the natural history of PAH or CTEPH over 6 months.

Methods

Conclusions 